\title{
A real-time EMG-driven musculoskeletal model of the ankle
}

\author{
Kurt Manal · Karin Gravare-Silbernagel • \\ Thomas S. Buchanan
}

Received: 16 June 2011 / Accepted: 2 November 2011 / Published online: 23 November 2011

(C) The Author(s) 2011. This article is published with open access at Springerlink.com

\begin{abstract}
The real-time estimation of muscle forces could be a very valuable tool for rehabilitation. By seeing how much muscle force is being produced during rehabilitation, therapists know whether they are working within safe limits in their therapies and patients know if they are producing enough force to expect improvement. This is especially true for rehabilitation of Achilles tendon ruptures where, out of fear of overloading and causing a rerupture, minimal therapy is typically done for eight weeks post-surgery despite animal studies that show that low-level loading is beneficial. To address this need, we have developed a biomechanical model that allows for the real-time estimation of forces in the triceps surae muscle and Achilles tendon. Forces are estimated using a Hill-type muscle model. To account for differences in neuromuscular control of each subject, the model used EMGs as input. To make this clinically useful, joint angles were measured using electrogoniometers. A dynamometer was used to measure joint moments during the model calibration stage, but was not required during real-time studies. The model accounts for the force-length and force-velocity properties of muscles, and other parameters such as tendon slack length and optimal fiber length. Additional parameters, such as pennation angle and moment arm of each muscle in the model, vary as functions of joint angle. In this paper, the model is presented and it application is demonstrated in two subjects: one with a healthy Achilles tendon and a second 6 months post Achilles tendon rupture and repair.
\end{abstract}

Keywords Muscle force $\cdot$ Joint moment $\cdot$ Multibody dynamics $\cdot$ Rehabilitation $\cdot$ Feedback

K. Manal · T.S. Buchanan $(\bowtie)$

Delaware Rehabilitation Institute, University of Delaware, Newark, DE, USA

e-mail: buchanan@udel.edu

K. Manal

e-mail: manal@udel.edu

K. Gravare-Silbernagel

Department of Physical Therapy, University of the Sciences, Philadelphia, PA, USA

e-mail: k.silbernagel@usciences.edu 


\section{Introduction}

Biofeedback is widely used in therapeutic interventions for rehabilitation of neuromuscular injuries. Although biofeedback of many types of information has been provided, one that has not been employed is information about the forces in muscles. There are two primary reasons for this. First, accurate estimation of muscle forces requires complex models and such models can be very time consuming computationally [1] making real-time estimation difficult. Second, the neural control of muscles in people with neuromuscular pathologies is different than that of healthy people, so many "generic healthy normal" biomechanical models cannot provide subject-specific estimations of muscle forces for those in need of such clinical interventions [2].

In this study, the joint of interest is the ankle. The soleus and gastrocnemii are the dominant ankle plantar flexors and share a common tendon distally: the Achilles. The Achilles is the largest tendon in the human body and the triceps surae (i.e., gastrocnemii and soleus) are the strongest muscles of the lower leg. The ability to predict triceps surae force and determine how individual muscles contribute to the plantar flexion moment is critical for the study of function and energetics during normal and pathological gait.

Achilles tendon injuries heal slowly and are often accompanied by residual weakness and decreased function [3]. Generally, patients with Achilles tendon ruptures are prevented from loading the ankle for a period of about 8 weeks to avoid the possibility of rerupture [4]. While it has been suggested that loading at an earlier stage may improve healing and longterm outcomes based on observations from animal models research indicates that improved outcomes might be achieved through controlled and progressive loading during the acute phase of therapy $[5,6]$.

By providing real-time estimations of triceps surae muscle forces and Achilles tendon force during specific physical therapy interventions, it will be possible to monitor the forces to make sure the patient's tendon is not being overloaded. It will also be possible to use this to establish a dose-response relationship, thereby determining the optimal therapies for applying the proper amount of force (i.e., dose) at the proper time during the rehabilitation process.

Numerous methods have been used for estimating muscle forces. We will use a musculoskeletal model driven by electromyograms (EMGs). An advantage of this approach is that magnitude and timing of subject specific neural activation patterns are used by the model since recorded EMGs are model inputs. Furthermore, muscle coactivation, which is common in those with pathology, is accounted for explicitly in the EMGs and should be taken into account if muscle forces are used to predict loading of musculoskeletal structures.

The question of how best to estimate Achilles tendon force in real-time is critical given the sensitive nature of the tendon during this phase of rehabilitation. The purpose of this paper is to present our EMG-driven modeling approach and data processing framework that allows us to predict Achilles tendon force in real-time. A demonstration of the clinical utility of this work will be presented by the estimation of Achilles tendon forces in two patients: one with a healthy Achilles tendon and a second six-months following Achilles tendon rupture.

\section{Methods}

Predicting muscle forces in real-time is achieved in a two-step process: (1) Tuning of an EMG-driven model, and (2) real-time implementation and graphical presentation of the model predicted forces. This section outlines these steps, briefly describes the experimental protocol used for model tuning and controlled testing, and also provides basic information for the two subjects tested. 


\subsection{Subjects}

Exemplar data from two subjects are presented to illustrate our EMG-driven modeling approach and application of the model in real-time. Subject 1 was a 40 year-old male who suffered an Achilles tendon rupture while playing soccer. The subject was 1.78 meters tall and weighed $73.5 \mathrm{~kg}$ at time of testing. Data were collected at 6 months following surgical repair. The subject performed 3 maximum voluntary isometric plantar flexion and 3 dorsiflexion contractions with the ankle at $0^{\circ}$ (i.e., neutral angle). Data were collected and EMGs recorded for the healthy and the surgically repaired legs. Optimal fiber length and maximum isometric force for soleus were based on average values calculated for subjects of similar stature. Subject 2 was a 45 year-old male $(1.78$ meters and $82 \mathrm{~kg}$ ) with no history of Achilles tendon injuries. The subject participated in a previous study in which optimal fiber length and maximum isometric force for the soleus, and the Achilles tendon moment arm were determined. These values were used during off-line tuning of the EMG-driven model. Only the left leg was tested for this subject. The subject also performed free-standing single and double leg quasi-dynamic tasks including heel-rises, squats, and closed-chain isometric plantar flexion while standing.

\subsection{Model tuning}

Our EMG-driven modeling approach is based on a Hill-type muscle model that has been previously described $[2,7]$. The model can be visualized as a muscle fiber in series with an elastic or viscoelastic tendon and a parallel elastic component to account for passive forces. The force developed by the muscle is related to the magnitude of the neural input (i.e., EMG) and the current state of the fiber (length and contraction velocity). Passive forces are developed in proportion to length relative to an optimum length (i.e., the length at which maximum isometric force is developed). Active and passive forces are balanced by tendon with tendon strain and force determined relative to a resting length (i.e., tendon slack length). Optimal fiber and tendon slack lengths are parameters required for each muscle in our EMG-driven model and these values vary between muscles and subjects. The ankle model presented in this paper consists of four muscles: (1) medial gastrocnemius (MG), lateral gastrocnemius (LG), soleus (Sol) and tibialis anterior (TA), and thus four values for optimal fiber and tendon slack length respectively are required. Idealized muscle parameters for each subject are determined using a combination of experimental testing and numerical optimization. We have developed methods to approximate soleus optimal fiber length and maximum isometric force in vivo, and a technique for measuring Achilles tendon moment arm. The methods combine video-based motion capture and ultrasonography to compute the distance from the ankle joint center to the Achilles tendon line of action, and also to measure soleus fiber length when isometric plantarflexion force is maximal $[8,9]$. Ultrasonongraphy is also used to measure fiber pennation angles for the triceps surae and tibialis anterior [10]. Once subject specific values for soleus optimal fiber length and pennation angle are determined they are used in combination with numerical optimization to estimate optimal fiber and tendon slack length for the gastrocnemii and TA. A simulated annealing algorithm is used to select optimal fiber lengths for the gastrocnemii and tibialis anterior, and also to obtain idealized values for tendon slack length for the plantarflexors and the tibialis anterior. 
These values were selected with the objective of minimizing the sum-squared difference between the model-estimated joint moment and the net moment recorded from dynamometry and/or inverse dynamics.

An advantage of using subject specific values for soleus (optimal fiber length and maximum isometric force) and EMGs is that it ensures a physiologic distribution of forces between the individual muscles of the triceps surae. That is, it prevents unrealistic solutions in which a single muscle generates the entirety of the plantar flexion moment. To enforce this, constraints were implemented using penalty functions to minimize occurrences of negative tendon strain (i.e., tendon length less than slack length) and to ensure normalized fiber lengths (fiber length/optimal fiber length) were within reasonable ranges (i.e., normalized fiber lengths between 0.6 and 1.2). Negative tendon strain and/or normalized fiber length less than 0.5 yield zero muscle force, while normalized lengths greater than 1.2 yield unrealistically large passive forces. Both scenarios are not physiologic considering the experimental setup (Fig. 1).

Optimization was also used to determine a strength gain coefficient for the plantarflexor muscle group and one for the TA. The same gain was applied to all plantarflexors and thus one muscle could not become disproportionally stronger than other muscles within the group. The gain coefficients were initially set to 1.0 and allowed to vary between 0.5 and 2.0 to accommodate individuals of differing strength.

The transformation from EMG to muscle activation (i.e., muscle activation dynamics) requires 4 additional parameters per muscle. These were selected by the optimizer and included a term for electro-mechanical delay ( $<70 \mathrm{~ms})$, a non-linear shape factor [11], and 2 coefficients defining a recursive filter. This transformation has been described in detail by Buchanan and colleagues [7].

Model-tuning for both subjects in this study used joint moment data collected from a dynamometer (Biodex Inc.). Ankle plantar and dorsiflexion moments were sampled at a rate of $1000 \mathrm{~Hz}$ and synchronized with EMGs recorded from the MG, LG, Sol, and TA (Fig. 1). The EMG signals were high-passed filtered $(30 \mathrm{~Hz})$ using a 4th order Butterworth digital filter, rectified and then low-pass filtered at a cut-off frequency of $4 \mathrm{~Hz}$. The filtered EMG signals for all trials were scanned to identify absolute peak values for each muscle and used to normalize all subsequent signals resulting in values ranging between 0 and 1 . The normalized and filtered EMGs were used as inputs to the EMG-driven model with musculotendon length and moment arms computed as a function of ankle and knee joint angles.

The bones of the lower leg model published by Delp and colleagues [12] were scaled to match individual subject specific segment lengths. Muscle attachment points scale with the bones and therefore subject specific musculotendon lengths are a function of the subject's ankle and knee kinematics. Achilles tendon moment arms were measured using a hybrid technique combining video-based motion capture and ultrasonography [9]. The moment arm for the TA was estimated using tendon excursion in the same manner as implemented in SIMM and OpenSim.

Subject 1 (6 months post-surgery) performed isometric plantar and dorsiflexion maximum voluntary contractions (MVCs) with the ankle in a neutral position ( 0 degrees) and the knee in 10 degrees of flexion. The second subject performed a more extensive series of Biodex trials including isometric MVCs, isokinetic MVCs at 30 degrees/second and several submaximal isokinetic exercises in both the plantar and dorsiflexion directions. The dynamometer trials used for model-tuning were processed off-line to determine a set of muscle parameters for each subject. A detailed description of the model tuning is presented elsewhere [7]. 
Fig. 1 Subject positioning in the Biodex. EMGs for the gastrocnemii, soleus and anterior tibialis were sampled at $1000 \mathrm{~Hz}$ and synchronized with the dynamometer data

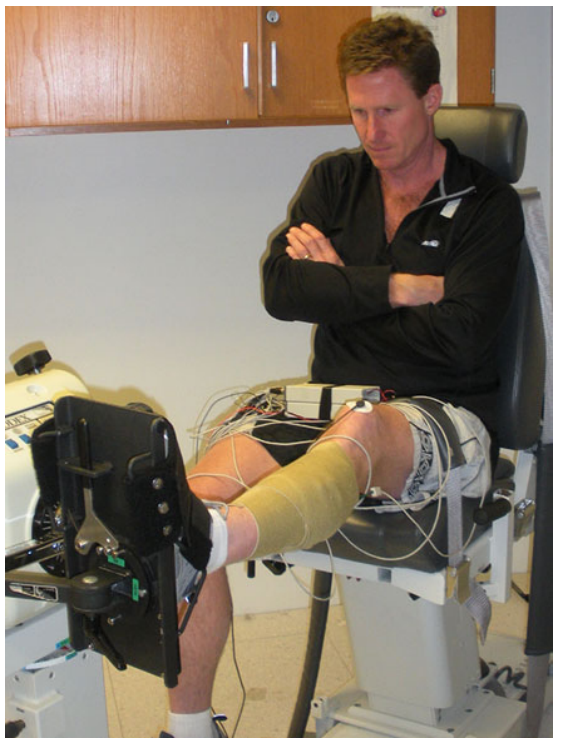

\subsection{Real-time modeling \& data visualization}

An integral component of our modeling framework is an efficient implementation of our EMG-driven musculoskeletal model. The software is multi-threaded with separate processes for data acquisition, kinematic and force calculations, and graphical display of a threedimensional model of the leg. Implicit in our model is that fiber and tendon forces must be balanced at each time step. This is because the fiber and tendon are in series and, therefore, the fiber force adjusted for pennation angle should equal the force carried by the tendon. This is solved under isometric conditions by iteratively adjusting fiber and tendon length for a given musculotendon length at each time step until the force in the fiber and tendon converge (i.e., within $0.1 \%$ of maximum isometric force potential of the muscle). Fiber force is dependent on fiber length while tendon force is a function of strain relative to its slack length. Although this simplification ignores fiber velocity effects, they can be approximated by scaling the isometric force by normalized musculotendon velocity computed from successive joint positions. Computing muscle force under isometric conditions as described herein is much faster than integrating fiber velocity to obtain fiber length. We do integrate fiber velocity when computing muscle forces off-line for more dynamic movements such as gait and have found that an adaptive step-size Runge-Kutta-Fehlberg integrator generally works well. Thus, the real-time version of our EMG-driven model is best suited for isometric contractions and slow quasi-dynamic movements such as those presented in this paper.

A three-dimensional graphical model of the lower limb and ankle muscles is displayed on a computer screen providing the subject with a visual representation of their leg. EMG, dynamometer and data from two electrogoniometers are sampled synchronously and acquired in blocks of 40 samples at a sampling frequency of $1000 \mathrm{~Hz}$ per channel. The analog data are filtered in real-time using a low pass Butterworth filter [13] with average signals over the 40 sample interval used for calculations. The model and the screen are updated 25 times per second providing real-time feedback of muscle forces and skeletal kinematics.

The real-time functioning of the model is verified by comparing the model predicted ankle moment with the moment recorded from the Biodex. Bar graphs (see Fig. 4) are used 
Table 1 Peak plantarflexion moments within a limb were similar as noted by the small standard deviations reported in parentheses. Achilles tendon force was obtained from the one trial used for model tuning and therefore no standard deviations are reported. The subject exhibited significant strength deficits and reduced Achilles tendon force 6 months post-op

\begin{tabular}{llll}
\hline & Uninjured & AT ruptured & Diff. (\%) \\
\hline Moment $(\mathrm{Nm})$ & $88.0(4.2)$ & $59.0(2.8)$ & -33.0 \\
AT force $(\mathrm{N})$ & 2310 & 1710 & -26.0 \\
\hline
\end{tabular}

to display the magnitude of the moment in real-time. When calibrated the Biodex moment (light bar) and the model-predicted moment (dark bar) should be of similar magnitude and change in the same direction. A more rigorous inspection of the data is also conducted off-line to verify the accuracy of the model (see Fig. 5). Once real-time verification of the model has been completed the subject is removed from the Biodex and free to perform quasidynamic exercises. Muscle forces during these tasks are predicted directly from EMGs and musculotendon lengths calculated based on joint angles recorded by the electrogoniometers. Triceps surae forces corrected for pennation angle are summed to approximate the Achilles tendon force and displayed in real-time using a bar graph for visualization (see Fig. 6).

\section{Results}

Subject 1: Strength at 6 months post-op The subject performed two MVC isometric plantar and dorsiflexion efforts with the ankle in a neutral position. It was evident during testing he attempted to contract maximally in both directions and for each leg. This is an important consideration when making side-to-side strength comparisons to minimize potentially confounding effects of differing effort. The average peak moment for the 3 plantar flexion trials recorded by the Biodex and the model-tuned Achilles tendon force (1 trial) are summarized in Table 1.

The results of model-tuning are shown in the top left and right panel of Fig. 2. Notice how the moment for the EMG-driven model oscillates about the smoother profile measured by the Biodex. The EMG-model converged nicely with high $R^{2}$ values for the uninjured $\left(R^{2}=0.987\right)$ and the surgically repaired tendon $\left(R^{2}=0.959\right)$. Notice how the pattern and timing of the ankle moments for the tuned model followed the pattern and timing of the moment recorded by the dynamometer. The bottom panel of Fig. 2 shows the model estimated Achilles tendon force for the uninjured and the Achilles tendon ruptured leg. Also included in the figures are the individual muscle force contributions of the triceps surae and TA.

Subject 2: Real-time implementation and data visualization Data for this subject are presented to demonstrate how the EMG-driven model can be used in real-time to monitor Achilles tendon force. The first step involved tuning the EMG-driven model to data collected from the dynamometer. The results of model tuning to a maximum isometric plantar and dorsiflexion trial are shown in Fig. 3. The subject was instructed to perform two cycles of time varying plantar and dorsiflexion efforts within the duration of the trial. The EMG-driven model successfully converged to the larger of the two plantar flexion peaks. The shapes of both curves were in close agreement as indicated by an $R^{2}$ value of 0.952 . Included at the 

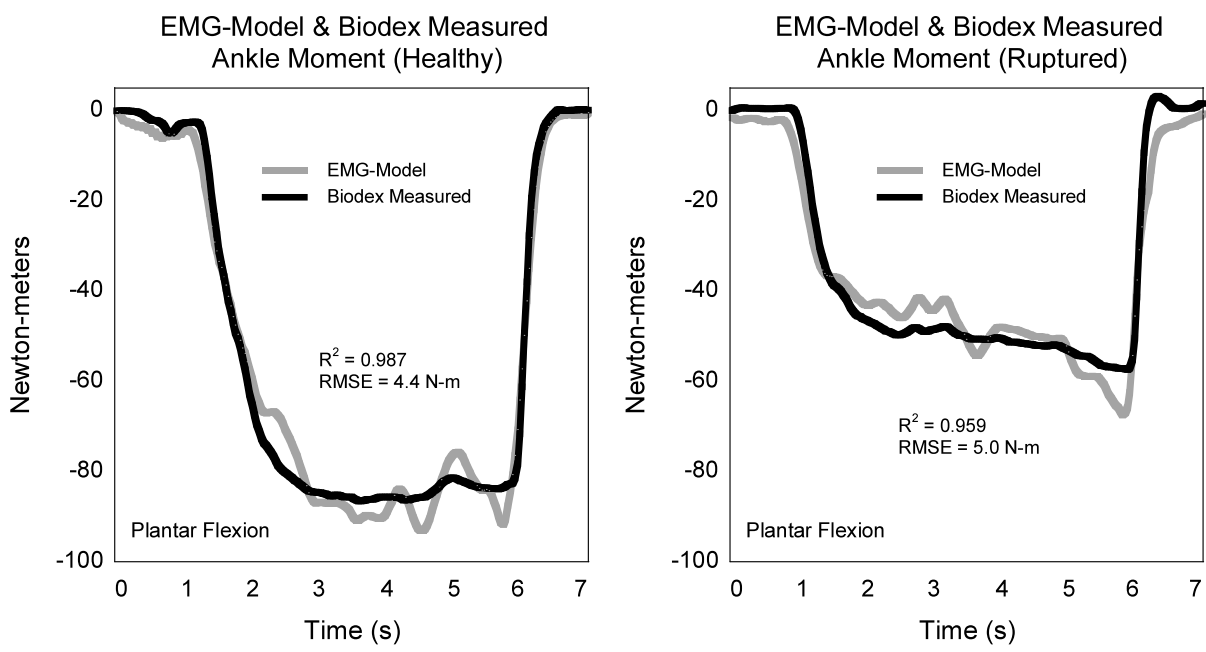

Triceps Surae \& Achilles Force (Healthy)

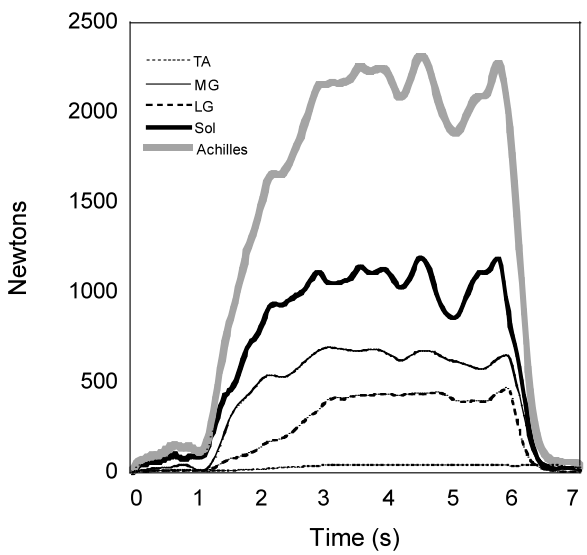

Triceps Surae \& Achilles Force (Ruptured)

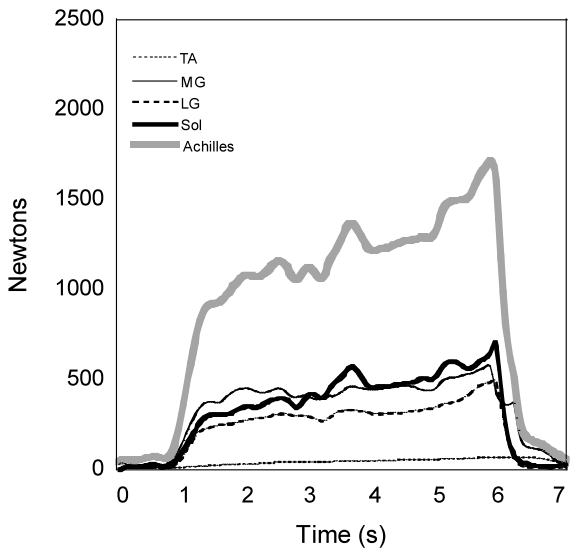

Fig. 2 (Top left) Maximum voluntary isometric plantar flexion moment for the uninjured leg (healthy) as measured by the dynamometer (Biodex Measured) for subject 1. The EMG-driven model was tuned to the plantar flexion moment and exhibited excellent agreement in both magnitude $\&$ timing (RMSE $=$ root mean square error). Individual muscle forces for the triceps surae, tibialis anterior and the resultant Achilles tendon force (bottom left panel) for the healthy leg. These forces contributed to the EMG-model plantarflexion moment shown in the top left panel. Peak plantar flexion moment was approximately $30 \%$ less for the ruptured side (top right panel) compared to the healthy leg. Peak triceps surae forces were smaller and more evenly distributed (bottom right panel) relative to the contra-lateral leg. The EMG-model converged nicely during model tuning $\left(R^{2}=0.959 \& \mathrm{RMSE}=5.0 \mathrm{Nm}\right.$ ). Tibialis anterior muscle force was less than $100 \mathrm{~N}$ indicating minimal co-contraction during the plantarflexion trials

bottom of Fig. 3 is the time-synchronized Achilles tendon force determined from the model. Peak Achilles force for the subject was approximately $3350 \mathrm{~N}$ and occurred at the same time as the peak plantar flexion moment. Interestingly, note how the model estimated a tendon force of approximately $500 \mathrm{~N}$ even when the net moment was in dorsiflexion.

After model-tuning is completed off-line, the model can be evaluated in real-time by comparing the Biodex measured moment with the moment computed by the model. This 
Fig. 3 Time-varying plantar (negative direction) and dorsiflexion moments during isometric MVC for subject 2. Excellent convergence during model tuning was achieved, especially for the second plantar flexion peak.

( $\mathrm{RMSE}=$ root mean square error). The model-estimated Achilles tendon force and time-line is shown at the bottom of the figure. Note how peak Achilles force coincided with the peak plantar flexion moments. Also note the model indicates the tendon remained loaded even when the net moment was in dorsiflexion

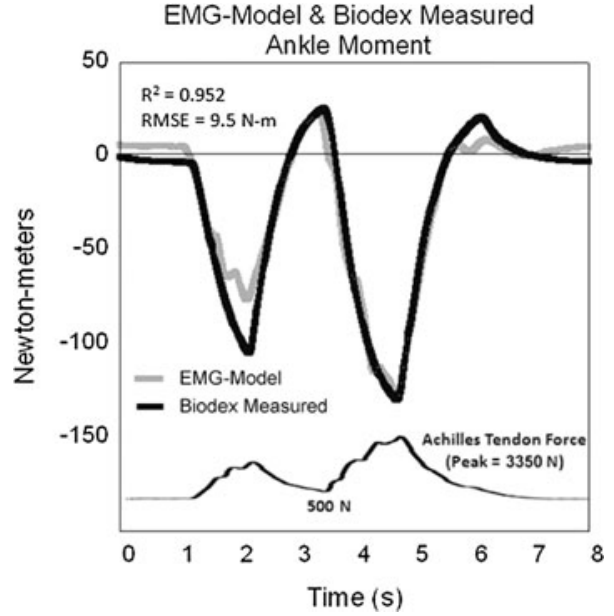

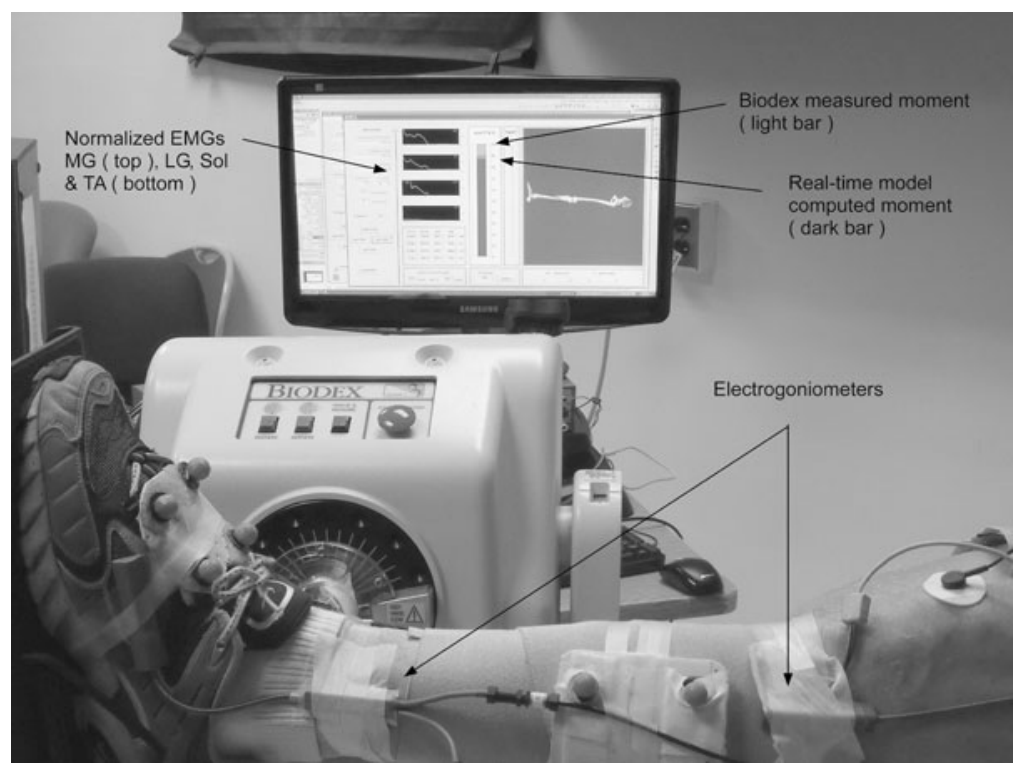

Fig. 4 Subject positioning in the dynamometer with the rotation axis aligned through the malleoli. Ankle and knee angles are recorded using electrogoniometers and used to orient the graphical model of the leg displayed on the computer screen. The light bar graph indicates the magnitude of the moment measured by the Biodex while the dark bar shows the moment predicted by the EMG-model and used to evaluate the model in real-time. The bars graphs should be of similar magnitude and move in the same direction when the EMG-model is calibrated. Normalized EMGs are also displayed to ensure quality of recordings

is shown in Fig. 4 using simple bar graphs to depict the magnitude of the moments. The light (Biodex moment) and the dark bar (EMG-model) should be of similar magnitude and change in the same direction when the model is calibrated and working well in real-time.

A more rigorous evaluation is also conducted to ensure the real-time model is behaving properly. This is done off-line. The real-time data are stored in memory and written to disk once data acquisition is complete. Model predictions are then compared to the ankle mo- 
Fig. 5 The EMG-model was used to predict ankle moments during a slow moving trial (30 degs/s) at sub-maximal effort. Note the magnitude of the peak moment $(-50 \mathrm{~N} \mathrm{~m})$ for this trial was significantly smaller than the trial used for tuning (Fig. 3, peak $=-130 \mathrm{Nm}$ ). The shape and timing of the model-predicted moment agreed closely with the moment recorded by the Biodex

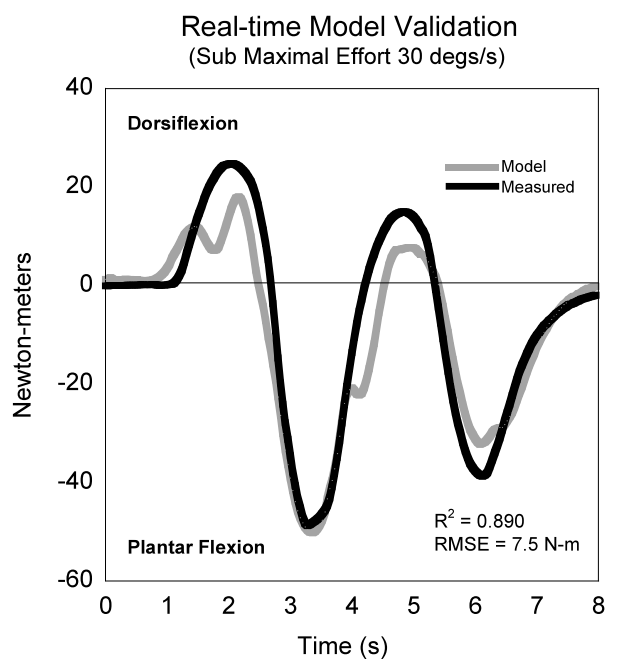

Fig. 6 After the EMG-model was tuned and the real-time moment predictions were verified during controlled testing (e.g., real-time dynamometer \& EMG-model moment comparisons), the subject was free to perform free-standing quasi-dynamic exercises.

Electrogoniometers were used to record joint angles and used to orient the graphical model of the leg on the computer screen. Note the orientation of the graphical model and the subject's actual leg are similar which is important for the real-time estimation of musculotendon length. The light bar graph was used to indicate an upper limit of Achilles tendon force with the dark bar showing the current level of force

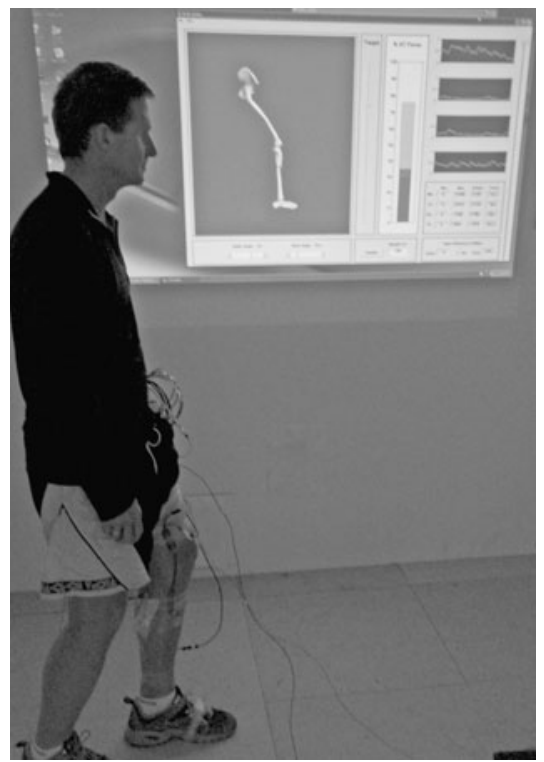

ment recorded by the dynamometer as illustrated in Fig. 5. Note the excellent agreement was not the result of retuning the EMG-driven model, but rather the moment was predicted from previously tuned muscle parameters, newly recorded EMGs and ankle angle dependent musculotendon lengths \& moment arms for the submaximum isokinetic trial.

The last example we present is intended to illustrate how Achilles tendon force can be monitored in real-time during slow quasidynamic tasks during free standing exercises. Electrogoniometers record ankle and knee joint angles which are used to compute musculotendon lengths and moment arms. Note how the leg of the graphical model shown in Fig. 6 is similar to the subject's actual limb configuration. Correct mapping is important for realistic musculotendon length estimates and therefore subsequent force calculations. The light and dark bar graphs previously used to display joint moment can now be used to visual- 
ize Achilles tendon force in real-time (dark bar) relative to a predefined upper limit shown by the light bar. For example, peak Achilles force during normal walking is approximately $1400 \mathrm{~N}$ [14]. In our example the upper limit force (light bar) in Fig. 6 was set at 80\% of 1400 $\mathrm{N}$. The subject was then instructed to move around, stand on $1 \mathrm{leg}$, squat and isometrically plantar flex while standing as long as the force level indicated by the dark bar remained below the prescribed limit of $80 \%$.

\section{Discussion}

The purpose of this paper was to describe our EMG-driven model for estimating muscle and tendon forces in real-time. Data from two subjects were presented to demonstrate the clinical utility of the modeling approach and the potential for real-time applications to guide rehabilitation following injury.

It is well documented that patients following Achilles tendon rupture have prolonged and often profound strength deficits. This was noted for subject 1 who at 6 months post-op had a $30 \%$ deficit in his ability to generate maximum plantar flexion moment. Patients tend to regain strength for up to 1 year following surgery however the long term prognosis is that a strength deficit of approximately 20-30\% will continue to persist [3].

It is important to consider potential risks when evaluating strength during MVC, especially after a major injury such as a complete Achilles tendon rupture. Our modeling approach assumes that contract maximally during MVCs, and thus if they are not capable of doing so the modeling results may be affected. This is an important consideration as maximal volitional contraction is used for EMG normalization and to determine maximum plantarflexion strength. The general consensus after Achilles tendon repair is that patients can return to full contact sports at 20-24 weeks after surgery [15], and MVC strength testing can be evaluated at 6 months post-op $[16,17]$. The patient in our study underwent surgery 6 months prior to testing and was fully cleared to participate in testing and did so without limitation and apprehension.

Recent research suggests that increased strength and improved function may be achieved with early loading of tendon during the acute phase of rehabilitation ( $<8$ weeks). Although this has been shown in animal models, there is a reluctance to test this theory in humans for fear of rerupturing the Achilles. Developing safe loading guidelines for different phases of rehabilitation is complicated by the fact that safe limits have yet to be defined. Consequently, during the initial 8 weeks following surgery, the patient wears a cast or brace designed to shield the tendon from excessive load. When the cast/brace is removed the patient is encouraged to start walking. The Achilles carries significantly load during gait (approximately $1400 \mathrm{~N}$ ), yet it is never exposed to loads approaching this magnitude during rehabilitation prior to return to walking. Perhaps a more effective training protocol would be to expose the tendon to increasing load as therapy progresses leading up to forces experienced during walking.

How best to estimate Achilles tendon force is a matter of debate. However, we suggest that simply dividing the net moment by the Achilles tendon moment arm should be avoided. For example, simply dividing the joint moment by the moment arm would predict zero loading whenever a dorsiflexion moment is present. In contrast, our model predicted the Achilles remained loaded (see Fig. 3), even when the net moment was in dorsiflexion. Tendon loading is to be expected for several reasons including (1) the triceps surae remain active (i.e., EMG > 0) even as the ankle moves into dorsiflexion, (2) the tendon has a force-dependent history (it does not go to zero instantaneously), and (3) the triceps surae is stretched when dorsiflexed and, therefore, develops passive forces. 
The EMG-driven model presented in this paper computes individual muscle forces in real-time and therefore it is an ideal tool for monitoring Achilles tendon loading during rehabilitation. The model can be used to quantify force during different exercises performed at various intensities. Since the model is driven by recorded EMGs, it is inherently sensitive to muscle cocontraction which is not uncommon in those with pathology. Subject 1 exhibited approximately twice the TA activity during maximum plantar flexion for the leg with the ruptured tendon compared to the uninjured side ( $11.7 \%$ vs. $6.5 \%$ of maximum TA activity). Coactivation of this magnitude would underestimate the Achilles tendon force by as much as $100 \mathrm{~N}$ based on model estimates if the TA had not been included in the model. Whether this difference is sufficient to pose an increased risk of injury is uncertain, however recall the subject was 6 months post-op at time of testing. Greater coactivation of TA might be expected earlier-on and possibly important to take into account when predicting tendon force in patients who coactivate their muscles.

Our real-time EMG-driven model is not without limitations. Although it is described as working in real-time, in reality every digital computer model has some computational delay and this model is no exception. There is an initial $40 \mathrm{~ms}$ delay during the first sampling of data and the initial force prediction, and a subsequent $2 \mathrm{~ms}$ delay per $40 \mathrm{~ms}$ acquisition interval associated with data processing. Musculotendon length is first calculated prior to computing muscle force and these steps have been arranged sequentially within a single thread of our multi-threaded application. Consequently, it is this step that requires the greatest amount of time. The delay, however, is not perceptible to the subject with the graphical model of the leg seemingly moving in concert with the subject. The software runs on a Dell Precision Workstation (2.8 GHz Xeon processor) and an ATI Radeon VE 7000 graphics adapter and therefore does not require specialized high-end hardware to work in "real-time."

Another assumption of our model is that the plantar flexion moment is developed entirely by the triceps surae. Although the gastroc-soleus complex is responsible for the vast majority, other muscles including the posterior tibialis, flexor hallucis longus, and the peroneals also contribute. Our model does not currently account for these muscles, however, it could be expanded to do so in the future. The consequence of not accounting for these muscles will be an overestimation of the actual force carried by the Achilles tendon. From a rehabilitation standpoint this is preferable compared to underestimating the force, especially when the model is used to monitor safe loading. That is, overestimating the force adds a built-in safety margin of sorts, and since the major ankle muscles are accounted for, this is not likely to be a substantial source of error.

Information about absolute and/or relative loading of the Achilles is useful for evaluating rehabilitation exercises following rupture and establishing criteria for progression. How best and safely to increase loading during acute rehabilitation is beyond the scope of this paper, however, we believe our modeling approach, especially the ability to monitor force in real-time may prove a useful tool in establishing dose-response relationships for optimum tendon healing while ensuring patients exercise within safe limits. Research that can result in specific guidelines on how to load the tendon during all the phases of healing to promote tendon healing and achieve early return of function would have a profound impact on the treatment and rehabilitation of all types of tendon injuries.

Acknowledgement This work is supported, in part, by NIH R01-AR046386.

Open Access This article is distributed under the terms of the Creative Commons Attribution Noncommercial License which permits any noncommercial use, distribution, and reproduction in any medium, provided the original author(s) and source are credited. 


\section{References}

1. Higginson, J.S., Neptune, R.R., Anderson, F.C.: Simulated parallel annealing within a neighborhood for optimization of biomechanical systems. J. Biomech. 38(9), 1938-1942 (2005)

2. Buchanan, T.S., Lloyd, D.G., Manal, K., Besier, T.F.: Estimation of muscle forces and joint moments using a forward-inverse dynamics model. Med. Sci. Sports Exerc., 37(11), 1911-1916 (2005)

3. Nilsson-Helander, K., Gravare-Silbernagel, K., Thomee, R., Faxen, E., Olsson, N., Eriksson, B.I., Karlsson, J.: Acute achilles tendon rupture: a randomized, controlled study comparing surgical and nonsurgical treatments using validated outcome measures. Am. J. Sports Med, 38, 2186-2193 (2010)

4. Suchak, A.A., Spooner, C., Reid, D.C., Jomha, N.M.: Postoperative rehabilitation protocols for Achilles tendon ruptures: a meta-analysis. Clin. Orthop. Relat. Res., 445, 216-221 (2006)

5. Enwemeka, C.S.: Functional loading augments the initial tensile strength and energy absorption capacity of regenerating rabbit Achilles tendons. Am. J. Phys. Med. Rehabil., 71, 31-38 (1992)

6. Aspenberg, P.: Stimulation of tendon repair: mechanical loading, GDFs and platelets. A mini-review. Int. Orthop., 31, 783-789 (2007)

7. Buchanan, T.S., Lloyd, D.G., Manal, K., Besier, T.F.: Estimation of muscle forces and joint moments and movements from measurements of neural command. J. Appl. Biomech. 20, 367-395 (2004)

8. Manal, K., Binder-Macleod, B., Buchanan, T.S.: Experimentally derived musculotendon parameters for the human soleus: Fiber length, pennation angle and isometric force. In: XXII Congress of the International Society of Biomechanics, Cape Town, South Africa (2009)

9. Manal, K., Cowder, J.D., Buchanan, T.S.: A hybrid method for computing Achilles tendon moment arm using ultrasound and motion analysis. J. Appl. Biomech. 26(2), 224-228 (2010)

10. Manal, K., Roberts, D.P., Buchanan, T.S.: Optimal pennation angle of the primary ankle plantar and dorsiflexors: variations with sex, contraction intensity, and limb. J. Appl. Biomech. 22, 255-263 (2006)

11. Manal, K., Buchanan, T.S.: A one-parameter neural activation to muscle activation model: Estimating isometric joint moments from electromyograms. J. Biomech. 36, 1197-1202 (2003)

12. Delp, S.L., Loan, P.J., Hoy, M.G., Zajac, F.E., Topp, E.E.T., Rosen, J.M.: An interactive graphics-based model of the lower extremity to study orthopaedic surgical procedures. IEEE Trans. Biomed. Eng., 37(8), 757-767 (1990)

13. Manal, K., Rose, W.C.: A general solution for the time delay introduced by a low-pass Butterworth digital filter: An application to musculoskeletal modeling. J. Biomech. 40, 678-681 (2007)

14. Finni, T., Komi, P.V., Lukkariniem, J.: Achilles tendon loading during walking: application of a novel optic fiber technique. Eur. J. Appl. Physiol., 77, 289-291 (1998)

15. Starkenburg, M., Donley, B., Dijk, Nv.: Guidelines for Sports resumption. Achilles tendon rupture. Current concepts: DJO publications, pp. 108-117 (2008)

16. Moller, M., Lind, K., Movin, T., Karlsson, J.: Calf muscle function after Achilles tendon rupture. A prospective, randomised study comparing surgical and non-surgical treatment. Scand. J. Med. Sci. Sports 12, 9-16 (2002)

17. Nilsson-Helander, K., Silbernagel, K.G., Thomee, R., Faxen, E., Olsson, N., Eriksson, B.I., Karlsson, J.: Acute achilles tendon rupture: a randomized, controlled study comparing surgical and nonsurgical treatments using validated outcome measures. Am. J. Sports Med. 38, 2186-2193 (2010) 